\title{
Homofilia e Assimetria na Rede de Coautoria de Proposições de Lei do Congresso Brasileiro
}

\author{
Lucas L. Rolim ${ }^{1}$, Daniel R. Figueiredo ${ }^{2}$ \\ ${ }^{1}$ Universidade Federal do Rio de Janeiro (UFRJ) \\ Escola Politécnica (POLI), Departamento de Engenharia Eletrônica (DEL) \\ Rio de Janeiro - RJ \\ ${ }^{2}$ Universidade Federal do Rio de Janeiro (UFRJ) \\ Programa de Engenharia de Sistemas e Computação (PESC) \\ Rio de Janeiro - RJ \\ lucasrolimepoli.ufrj.br, danielecos.ufrj.br
}

\begin{abstract}
Resumo. A Câmara dos Deputados é a instância máxima de representatividade do povo e uma de suas principais atividades é a proposição e aprovação das leis que regem o país. Entretanto, as 513 cadeiras da Câmara são ocupadas por deputados de diferentes estados, partidos, etnias e graus de escolaridade, sendo necessário articulações e alianças políticas na proposição e aprovação de leis. $O$ objetivo deste trabalho é caracterizar a rede de coautoria visando identificar homofilia e assimetria de diferentes atributos no processo de proposições de leis. Uma metodologia para aferir homofilia da rede e de indivíduos é proposta e avaliada, identificando diferentes aspectos relevantes, como a alta assimetria de diferentes categorias e a falta de homofilia expressiva entre as minorias.
\end{abstract}

\begin{abstract}
The Chamber of Deputies is the maximum degree of people representativity in Brazil, having as one of its main goals the approval of law and projects to develop and manage the country. We have deputies from different regions, parties, sex, ethnicity, and education levels occupying the 513 existing chairs and creating alliances and negotiations to approve their projects in this heterogeneous environment. The goal of this work is to describe the coauthorship network among these deputies, concentrating in identify and characterize homophily and asymmetry patterns. We will propose and evaluate a new methodology to analyze the homophily in the congress network. Using the proposed methodology we will identify important aspects, as a high level of asymmetry and a lack of homophily among minorities.
\end{abstract}

\section{Introdução}

A Câmara dos Deputados é a instância máxima de representatividade do povo no estado brasileiro, sendo o principal pilar do Poder Legislativo. A atividade fim desse órgão é, através dos seus membros (deputados), elaborar proposições de lei que visem discutir e aprimorar áreas econômicas e sociais, bem como fiscalizar o emprego, pelos Poderes da União, dos recursos arrecadados da população com o pagamento de tributos. 
Toda lei do estado brasileiro necessita passar por votação e aprovação da Câmara dos Deputados para se tornar vigente, bem como a maioria dessas são articuladas e desenvolvidas pelos membros da própria casa. Para a aprovação, uma proposição de lei precisa de apoio dos membros da Câmara, gerando assim a necessidade de articulações e alianças políticas dos agentes interessados.

O Brasil conta atualmente com 513 deputados federais, cada qual com direito a suplentes em caso de saída, que se renovam a cada quatro anos por meio de eleições diretas populares. As cadeiras da Câmara dos Deputados são distribuídas proporcionalmente de acordo com a população dos 26 estados membros da república federativa brasileira e o distrito federal. Além disso, 35 partidos diferentes ocupam diferentes quantidades de cadeiras na atual legislatura, possuindo ainda diferentes etnias e grau de escolaridade. Como tais características se relacionam com o processo de coautoria de proposições de lei? Deputados de um estado tendem a escrever projetos com deputados do mesmo estado? Esta tendência depende do estado?

Esta pergunta pode ser atacada através do estudo da rede de coautoria, onde vértices são deputados e arestas representam coautoria em proposições de lei. Tendo em vista que vértices possuem diferentes atributos, como partido e região, técnicas de caracterização de homofilia em redes podem ser utilizadas.

A homofilia pode ser caracterizada como a maior predisposição dos indivíduos de formarem relações baseados na similaridade entre suas características [Madan et al. 2011, Newman 2018]. Por exemplo, indivíduos que frequentaram a mesma universidade e possuem a mesma nacionalidade têm mais chance de ser amigos no Facebook [Seder and Oishi 2009]. Nesse contexto, podemos avaliar o grau em que agentes políticos estão suscetíveis a essa influência para cada uma das características coletadas.

A caracterização da homofilia da rede é portanto o objetivo deste trabalho, que faz as seguintes contribuições:

- Construção de um dataset unificado a partir de diferentes fontes públicas de informação com dados de deputados e proposições de leis. Este dataset está disponibilizado publicamente em um diretório na nuvem, assim como o software utilizado para construção do dataset está disponível no GitHub.

- Caracterização da rede de coautoria dos deputados na legislatura 55 (entre 20152018) levando em consideração diferentes atributos (partido, região, etnia, etc), e propondo métricas para calcular a homofilia da rede e de vértices.

- Estudo detalhado da homofilia da rede e de indivíduos de diferentes atributos, revelando diversas características do processo de proposição de lei. Em particular, a forte assimetria na homofilia indicando que alguns partidos ou estados ou etnias possuem muito mais homofilia do que outros. A mesma assimetria foi identificada em indivíduos.

Diversos trabalhos estudam redes formadas por congressistas, tanto do congresso brasileiro quanto de outros países (ver seção 2). Entretanto, no contexto brasileiro, não é de conhecimento dos autores um estudo da rede de coautoria de proposições de lei e consequentemente da homofilia e assimetria desta rede, sendo este um importante diferencial deste trabalho (mais detalhes na seção 2).

Uma das questões centrais no estudo e caracterização de homofila é a análise 
de minorias, como mulheres e negros no congresso brasileiro. Em particular se estes grupos tendem a se apoiar mais entre si do que aos demais parlamentares em média. Pesquisas anteriores apontaram que a identificação de um indivíduo com um subgrupo é mais forte quando esse subgrupo é uma minoria [Huddy 2004]. Observaremos que este fenômeno não ocorre no congresso brasileiro como um todo, mas que pode ocorrer com determinados deputados em particular.

Na seção 2 trabalhos relacionados são brevemente descritos. A seção 3 descreve o processo de criação da rede, bem como as premissas adotadas e suas limitações. A seção 4 apresenta uma caracterização de propriedades elementares desta rede. A seção 5 define as métricas de homofilia utilizadas assim como uma avaliação e discussão comparativa destes resultados. Por fim, a seção 6 encerra o artigo com uma breve conclusão.

\section{Trabalhos Relacionados}

Tendo em vista sua importância para o sistema político Brasileiro, a Câmara dos Deputados é objeto de diversos estudos ao longo dos anos, geralmente conduzidos de forma qualitativa no contexto de Ciências Políticas e Ciências Sociais [Miguel 2003, Nicolau 2002]. Mais recentemente, com a crescente disponibilidade e quantidade de dados, em parte por conta da Lei de Acesso à Informação, diversos estudos quantitativos de larga escala da Câmara dos Deputados vem sendo realizados [Baptista et al. 2014, Bursztyn et al. 2016, Ferreira et al. 2018, Levorato and Frota 2017, Miguel et al. 2015, Verona et al. 2018].

Alguns estudos quantitativos recentes da Câmara dos Deputados brasileira constroem redes onde os deputados são os vértices e analisam a rede em busca de um melhor entendimento de características do congresso [Baptista et al. 2014, Bursztyn et al. 2016, Ferreira et al. 2018, Levorato and Frota 2017, Verona et al. 2018]. Entretanto, todos estes trabalhos formam e analisam redes de co-votação, onde o relacionamento entre dois deputados (representado por uma aresta) é o mesmo voto em um ou mais projetos de lei. Uma exceção é a rede de co-doação, onde o relacionamento entre dois deputados é o recebimento de doação para a campanha eleitoral de um mesmo do doador [Bursztyn et al. 2016]. Estes trabalhos analisam diferentes aspectos da rede, como a formação de comunidades, e a importância ou influência dos deputados.

Diferente destes, o presente trabalho considera a rede de coautoria em proposições de lei, onde o relacionamento entre dois deputados representa a autoria de uma mesma proposição de lei. Esta rede é fundamentalmente diferente da rede de co-votação e codoação, sendo uma rede que codifica mais fielmente as relações sociais e profissionais entre os deputados. Desta forma, a análise desta rede irá revelar aspectos distintos da dinâmica política no congresso brasileiro, contribuindo para um melhor entendimento deste complexo sistema.

No contexto do congresso dos Estados Unidos da América, diversos estudos tratam da rede de cosponsorship onde um parlamentar declara seu apoio publicamente a um projeto de lei proposto por outro parlamentar, mesmo antes do projeto ser submetido à votação[Kessler and Krehbiel 1996, Fowler 2006]. Estas redes são inerentemente direcionadas, pois um deputado declara apoio a um outro (que por sua vez pode declarar apoio ao primeiro). Entretanto, redes de cosponsorship não são viáveis de serem construídas no contexto brasileiro, tendo em vista o sistema político nacional e as informações disponíveis publicamente. Apesar disto, a rede de coautoria considerada no presente tra- 
balho certamente está relacionada às redes de cosponsorship, pois a coautoria em uma proposição de lei pode ser vista como um apoio mútuo entre dois coautores da proposição.

\section{Criação da rede}

Os dados utilizados neste trabalho foram extraídos da API de Dados Abertos da Câmara dos Deputados e do Repositório de dados eleitorais do Tribunal Superior Eleitoral. Ambas são iniciativas de dados abertos do governo brasileiro, visando uma maior transparência sobre o funcionamento da máquina pública.

Em particular, foram extraídas informações sobre as proposições de lei criadas ou aprovadas em uma determinada legislatura, informações pessoais dos parlamentares dessa legislatura, informações oficiais de cada partido e também os cargos ocupados por cada um dos membros do congresso no intervalo de tempo definido. Tanto proposições de lei tramitadas quanto não tramitadas foram consideradas, com foco nas proposições do tipo Projeto de Emenda Constitucional e Projeto de Lei e suas variações. Em particular, foram extraídas e avaliadas 12.190 proposições de lei, cada qual com um determinado número de coautores. O conjunto de proposições de lei será representado por $\mathcal{P}$ e $\mathcal{C}_{p}$ representa o conjunto de coautores da proposição de lei $p \in \mathcal{P}$.

A figura 1 mostra a distribuição do número de coautores das proposições de lei. Vale notar que o número médio de coautores é de 1.95 , enquanto os valores mínimo e máximo são, respectivamente, de 1 e 37 . A distribuição indica que o número de coautores em proposições de lei possui cauda pesada, com algumas proposições contendo muito mais coautores que a média. A proposição com mais coautores é a PEC139/3391, que trata sobre a concessão ou não de anistia ao policial que coordenou a Operação Policial Satiagraha.

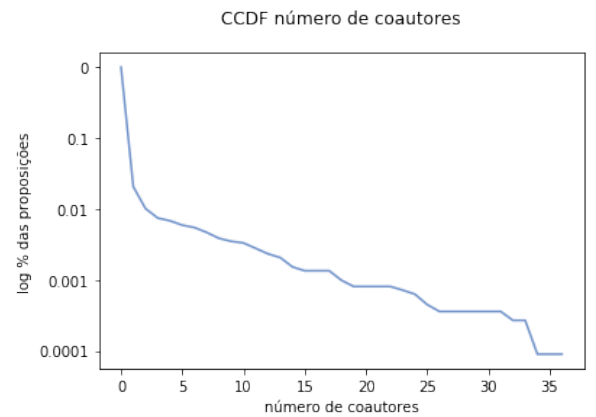

Figura 1. Nove proposições $(0,04 \%$ do total) possuem mais de 20 coautores

O conjunto de vértices da rede, representado por $\mathcal{V}$, representa os deputados federais que possuíram mandato ativo no intervalo escolhido. Desses deputados foram coletados os atributos disponíveis na API governamental e que comumente são utilizados para análise de redes sociais políticas, como os estudos que serão referenciados ao longo deste trabalho. Sendo assim, cada um desses vértices possui os atributos descritos na tabela 1 .

Cada proposição de lei $p \in \mathcal{P}$ dá origem a um clique no qual os vértices correspondem aos coautores da proposição, ou seja, $\mathcal{C}_{p}$. Desta forma, a rede é formada por uma união de cliques. Além disso, um determinado par de deputados pode ser coautor em mais de uma proposição de lei. Esta informação será codificada no peso da aresta, $w_{i, j}$. Em 
Tabela 1. Atributos coletados dos deputados

\begin{tabular}{|l|l|l|}
\hline Atributo & Tipo & Número de categorias \\
\hline Nome & Texto & - \\
\hline Idade & Inteiro & - \\
\hline Partido & Categórico & 27 \\
\hline UF & Categórico & 28 \\
\hline Escolaridade & Categórico & 6 \\
\hline Etnia & Categórico & 4 \\
\hline Sexo & Categórico & 2 \\
\hline
\end{tabular}

particular, podemos definir esse peso de aresta da seguinte forma:

$$
w_{i, j}=\sum_{p \in \mathcal{P}} \mathbb{1}\left(i, j \in \mathcal{C}_{p}\right)
$$

onde $\mathbb{1}(\cdot)$ é a função indicadora que retorna 1 se a condição é verdadeira e 0 caso contrário. Repare que $w_{i, j}=0$ se e somente se $i, j \in \mathcal{V}$ não foram coautores em um mesmo projeto de lei.

O grau do vértice $i$, definido como $d_{i}=\sum_{j \in V} \mathbb{1}\left(w_{i, j}>0\right)$ representa o número de coautores do deputado $i$ considerando todos as proposições de lei. Por fim, $w_{i}=$ $\sum_{j \in V} w_{i, j}$ denota o peso do vértice $i \in \mathcal{V}$ que representa o número de coautorias do deputado $i$ considerando todos as proposições de lei (contando um mesmo coautor mais de uma vez).

É importante ressaltar que outros trabalhos utilizam o conceito de cosponsorship para formação da rede de deputados, que neste caso é direcionada e as arestas representam o apoio formal que um congressista oferece a outro [Fowler 2006]. Este é o caso do congresso dos EUA, por exemplo. Todavia, esse tipo de abordagem é inviável no caso brasileiro devido a não discriminação explícita por entre os autores e coautores de uma proposição de lei. Assim sendo, todo trabalho foi desenvolvido sem a discriminação entre um autor e um coautor de um projeto de lei.

\section{Caracterização da Rede}

O estudo da estrutura da rede se faz importante para entender o contexto geral da interação entre deputados e estabelecer um panorama sobre a coautoria de projetos no Brasil. Além disso, um estudo inicial destas características permite um primeiro olhar sobre assimetrias e disparidades entre os parlamentares brasileiros.

Um primeiro aspecto interessante é o número de vértices presentes na rede. Ao contrário dos 513 vértices esperados, número de deputados federais previstos na Constituição, na legislatura 55 houve um total de 633 agentes que participaram do congresso. Esse maior número ocorre devido a posse de suplentes, causada por casos de parlamentares que abriram mão de sua posição para concorrer a outros cargos e também de parlamentares que tiveram seu mandato cassado. Essa discrepância mostra que, entre os anos de 2015 e 2018, aproximadamente $23 \%$ dos agentes envolvidos no desenvolvimento de proposições de lei não eram representantes eleitos diretamente pelo povo.

A figura 2 apresenta a distribuição de grau dos vértices, que apresenta uma cauda pesada (alguns vértices possuem grau muito maior do que a média). Note que cerca de 
Tabela 2. Propriedades da rede de coautoria.

\begin{tabular}{|l|l|}
\hline Característica & Valor \\
\hline Vértices, Arestas & 633,4985 \\
\hline Densidade & $2,49 \%$ \\
\hline Coeficiente de clusterização & 0.39 \\
\hline Tamanho da maior componente conexa (percentual) & $356(56 \%)$ \\
\hline Vértices com grau maior que zero & 360 \\
\hline Grau (menor, médio, maior) & $0,15,7,101$ \\
\hline Peso de aresta (menor, médio, maior) & $1,1,48,15$ \\
\hline
\end{tabular}

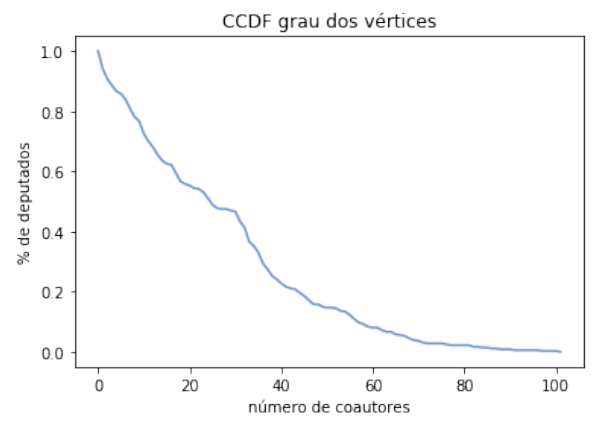

Figura 2. Distribuição empírica complementar cumulativa dos graus da rede.

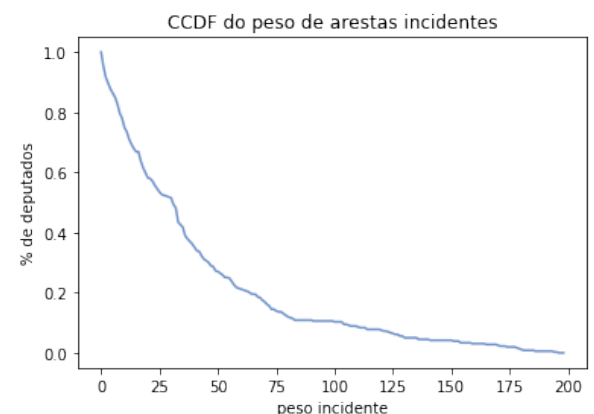

Figura 3. Distribuição empírica complementar cumulativa do peso dos vértices da rede.

$50 \%$ dos parlamentares tiveram menos de 20 coautores em projetos e destes $43 \%$ possuem grau zero. O maior grau da rede, de valor 101, pertence ao deputado Alberto Fraga, membro do partido DEM e representante do Distrito Federal. Esse parlamentar já ocupou os cargos de vice líder dos partidos PMDB, PFL e DEM, o que justifica sua posição de destaque. Esta análise ilustra a forte assimetria existente no processo de coautoria de proposições de lei desta legislatura.

A figura 3 apresenta a distribuição do peso dos vértices. Esta distribuição se assemelha à distribuição de grau, contando porém com uma cauda pesada mais acentuada. Observando essa métrica podemos notar que apenas 10\% dos deputados concentram 50\% dos pesos da rede. Ainda, entre os 5 vértices com maior peso (Evair de Melo, Vitor Lippi, Pedro Uczai, e João Henrique Caldas), todos que se candidataram foram reeleitos para mandato na legislatura 56 (2019-2022). Esse resultado permite uma primeira inferência sobre a existência de correlação entre a relevância do parlamentar no processo de coautoria e seu desempenho no processo eleitoral, correlação esta que poderá ser melhor investigada em trabalhos futuros.

\subsection{Estudo dos atributos da rede}

Com exceção do número de deputados por região, que possui número fixo definido com base na população de cada estado da federação, a maior parte dos atributos coletados apresentam grande assimetria em suas distribuições.

$\mathrm{Na}$ legislatura 55, dos 35 partidos existentes, apenas 5 (MDB, PT, PP, PSDB e $D E M)$ englobavam mais de $50 \%$ dos vértices da rede. Levando em consideração o peso incidente dos vértices essa concentração fica também evidente, com $41 \%$ do peso do grafo 
incidindo sobre vértices de apenas três partidos (PT, DEM, PSB). Além disso, é possível notar grande polarização entre os partidos DEM (oposição conservadora) e PT (partido governista de esquerda) por meio da visualização da rede apresentada na figura 4 (gerada pelo openOrd [Martin et al. 2011]).

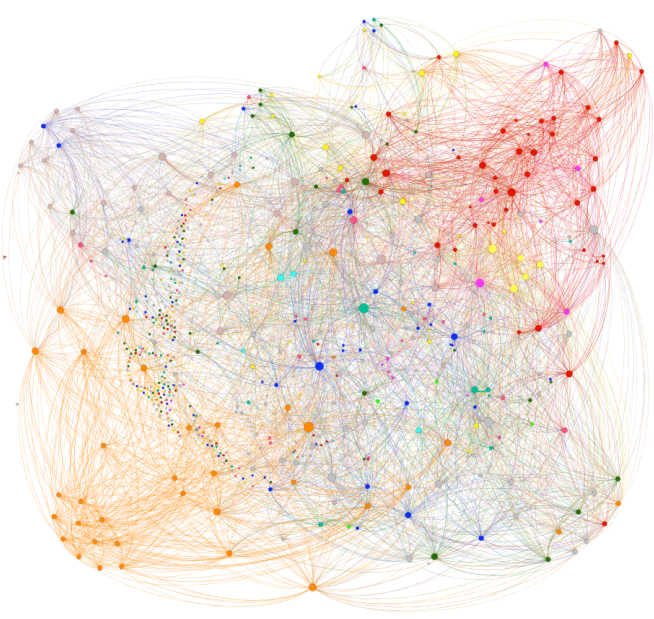

Figura 4. Rede de coautoria dos deputados com cores indicando partidos políticos (PT em vermelho e DEM em laranja).

Por fim, outras fortes discrepâncias entre a distribuição das características dos membros na Câmara e da população brasileira foram encontradas. Apesar de representarem $52 \%$ do eleitorado brasileiro, apenas $10 \%$ dos congressistas são do sexo feminino. De forma semelhante, negros e pardos somam aproximadamente $51 \%$ da população brasileira, porém compõem apenas $25 \%$ dos deputados atuantes nesta legislatura.

\section{Análise da homofilia}

A homofilia pode ser mensurada a partir de diferentes atributos dos deputados, tais como atributos pessoais como etnia, escolaridade e gênero, e atributos externos, tais como partido político e estado de origem. Não obstante, avaliaremos também a homofilia dos diferentes valores desses atributos.

\subsection{Métrica de homofilia}

Para mensurar a homofilia dos atributos da rede será utilizada a métrica de modularidade proposta por Newman [Newman 2018]. Esta métrica captura a homofilia utilizando a relação entre a fração de arestas com as duas pontas incidindo em vértices que possuem uma determinada característica e a fração de arestas com ao menos uma ponta incidindo em um vértice com esta mesma característica. Quanto menor for esta diferença, maior será a homofilia.

A métrica originalmente proposta não considera redes com peso e será adaptada neste trabalho pra este caso. Além disso, vértices nestes trabalho possuem diferentes atributos (ex. partido e sexo) que possuem diferentes valores (ex. Rio de Janeiro e Masculino, 
respectivamente), e a notação a ser introduzida irá refletir esta dimensão. Seja $\mathcal{A}$ o conjunto de atributos de cada vértice da rede. Seja $a \in \mathcal{A}$ um destes atributos, e defina $\mathcal{R}_{a} \mathrm{o}$ conjunto de valores que o atributo $a$ pode assumir. Seja $g_{a}(i)$ o valor do atributo $a \in \mathcal{A}$ do vértice $i \in V$, e repare que $g_{a}(i) \in \mathcal{R}_{a}$. funções:

Seja $a \in \mathcal{A}$ um atributo e $r \in \mathcal{R}_{a}$ um de seus valores. Defina as duas seguintes

$$
\begin{gathered}
x(a, r)=\frac{1}{2 W} \sum_{i \in V} \sum_{j \in V} w_{i, j} \mathbb{1}\left(g_{a}(i)=r\right) \mathbb{1}\left(g_{a}(j)=r\right), \\
y(a, r)=\frac{1}{2 W} \sum_{i \in V} \sum_{j \in V} w_{i, j} \mathbb{1}\left(g_{a}(i)=r\right),
\end{gathered}
$$

onde $\mathbb{1}(\cdot)$ é a função indicadora, que vale 1 quando a condição é verdadeira e 0 caso contrário, e $W=\sum_{i j} w_{i, j}$ a soma dos pesos das arestas da rede. Repare que $x(a, r)$ denota a fração de peso das arestas que tem suas duas pontas em vértices com o mesmo valor $r$ para o atributo $a$. Repare ainda que $y(a, r)$ denota a fração de peso das arestas que possuem ao menos uma ponta em vértices com o valor $r$ para o atributo $a$. Desta forma, temos que $x(a, r) \leq y(a, r)$.

Finalmente, a modularidade do atributo $a$ denotada por $Q_{a}$, é definida como:

$$
Q_{a}=\sum_{r \in \mathcal{R}_{a}}\left(x(a, r)-y(a, r)^{2}\right)
$$

Esta métrica relaciona o observado empiricamente na rede (valor de $x(a, r)$ ) com o valor caso as pontas de arestas fossem conectadas ao acaso (valor de $y(a, r)^{2}$ ) [Newman 2018].

\subsection{Homofilia na rede}

Conforme exposto pega figura 5 , os atributos de partido e região apresentaram uma influencia significativa na homofilia do processo de coautoria de proposições de lei, enquanto características como sexo, etnia e escolaridade se mostrarem praticamente irrelevantes.

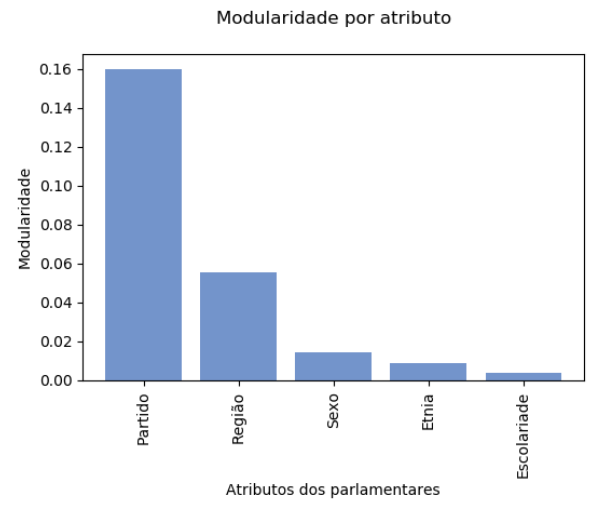

Figura 5. Medida de homofilia das características coletadas

Esse resultado permite inferir que, no cenário brasileiro, agrupamentos políticos ideológicos e regionais são capazes de sobrepor a predisposição apontada pela teoria social de minorias de colaborarem de forma mais significativa entre si. 


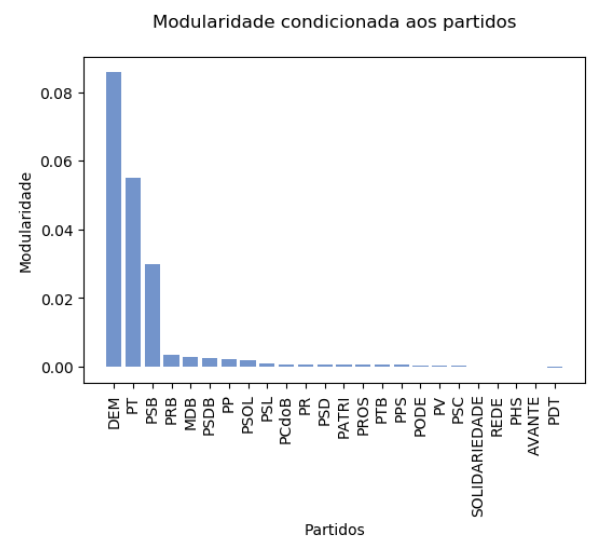

Figura 6. Modularidade dos partidos políticos

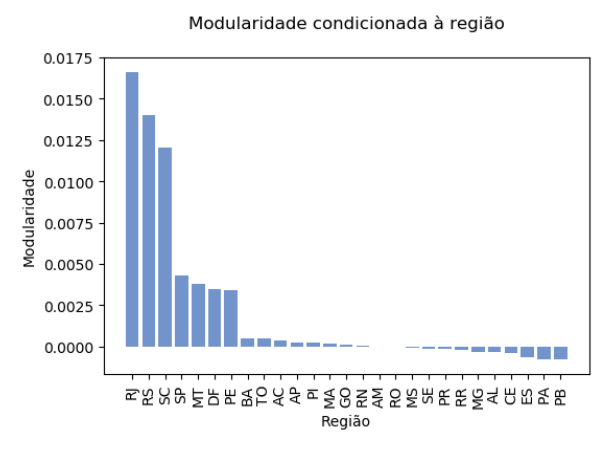

Figura 7. Modularidade das regiões

\subsection{Homofilia condicionada a atributos}

Dado que partido e região foram os atributos mais influentes em relação a homofilia, explorou-se a natureza inter grupo desses atributos de modo a descobrir como se comportam deputados de diferentes regiões e partidos.

Em relação aos partidos nota-se,conforme a figura 6, que a homofilia está concentrada principalmente em três partidos: DEM, PT e PSB. O destaque para os partidos DEM e PT são significativos pois podem ser interpretados como a existência de uma base coesa tanto no aspecto governista quanto no aspecto em um dos principais partidos de oposição.

Em relação ao atributo de região, destaca-se, como pode ser visto na figura 7,a homofilia na região Sul do país. Dois de seus três estados (RS e PR) possuem algumas das maiores homofilias da rede, além do fato de que a ligações entre seus três estados membros apresentam pesos médios de arestas maiores do que a rede de maneira geral.

\subsection{Homofilia individual dos vértices}

Visando o estabelecimento de uma métrica local de homofilia que possa ser atribuída aos vértices, definiu-se uma adaptação da fórmula de modularidade de Newman apresentada anteriormente. Intuitivamente, esta métrica compara a homofilia entre o vértice e seus vizinhos com a homofilia da rede. Desta forma, um vértice pode exibir uma homofilia maior ou menor do que a homofilia presente na rede como um todo.

Considere um atributo $a \in \mathcal{A}$ e um vértice $i \in V$. Defina a seguinte função:

$$
x^{\prime}(a, i)=\frac{1}{W_{i}} \sum_{j \in V} w_{i, j} \mathbb{1}\left(g_{a}(i)=g_{a}(j)\right),
$$

onde $W_{i}=\sum_{j} w_{i, j}$ é a soma dos pesos das arestas incidente ao vértice $i$. Repare que $x^{\prime}(a, i)$ denota a fração de peso que o vértice $i$ possui com vizinhos que tem o mesmo valor no atributo $a$.

A definição acima é comparada com a fração de peso da rede como um todo, dando o origem a seguinte definição para homofilia de um vértice:

$$
Q_{i, a}^{\prime}=x^{\prime}(a, i)-x\left(a, g_{a}(i)\right)
$$


Repare que $Q_{i, a}^{\prime}$ é um valor no intervalo $[-1,1]$ indicando que um vértice pode possuir uma homofilia mais forte ou mais fraca que a homofilia da rede no atributo $a$. Por fim, podemos definir a homofilia de um vértice considerando todos os atributos da rede, através da seguinte soma:

$$
Q_{i}^{\prime}=\sum_{a \in \mathcal{A}} Q_{i, a}^{\prime}
$$

Novamente, repare que $Q_{i}^{\prime}$ pode assumir valores positivos ou negativos, indicando se o vértice $i$ possui uma homofilia mais ou menos acentuada que a homofilia da rede.

A figura 8 apresenta a homofilia dos vértices segundo a métrica acima (ordenadas da menor para a maior). Nota-se que a grande maioria dos deputados apresenta baixo grau de homofilia, seja de forma negativa ou positiva, mas próximas a zero. Em particular, apenas $142(22 \%)$ apresentam significativa influência das características dos parlamentares no processo formação de coautores.

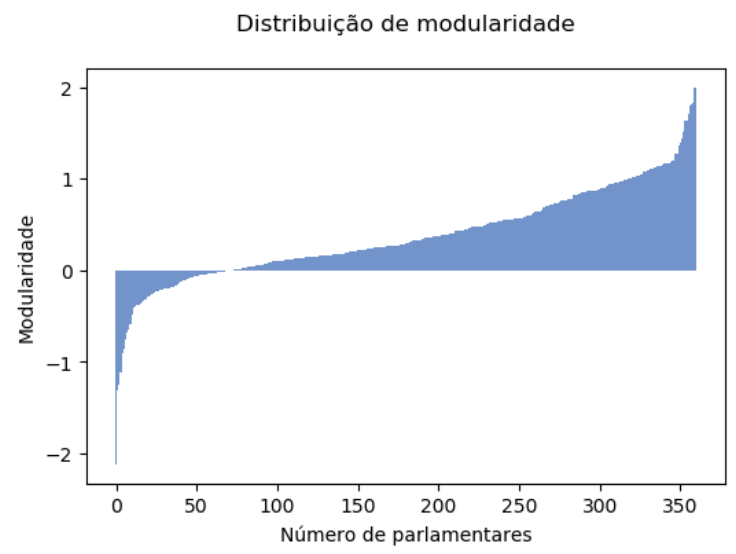

Figura 8. Distribuição da modularidade individual dos vértices (excluindo vértices de grau zero)

Condicionando essa distribuição aos atributos de região e partido, obtermos respectivamente as figuras 9 e 10. Nota-se que esses dois atributos, que figuram como os de maior influência homofílica da rede, possuem distribuições semelhantes e com muito menos parlamentares apresentando valores negativos ou insignificantes.

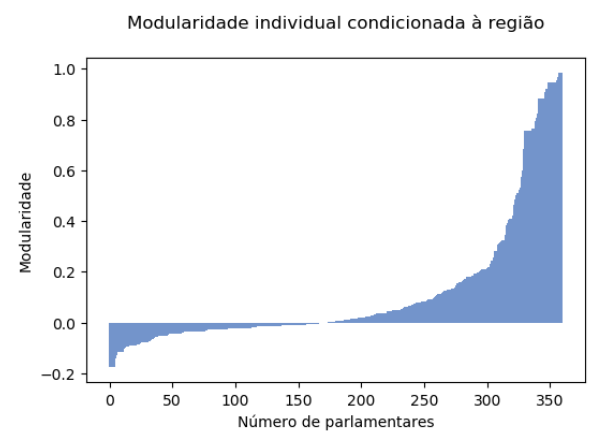

Figura 9. Distribuição de modularidade dos deputados condicionada à região

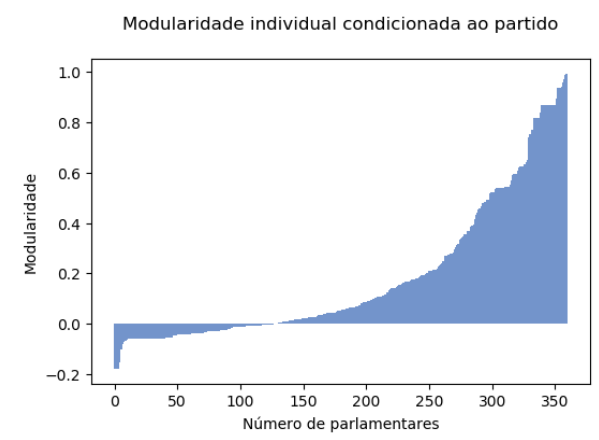

Figura 10. Distribuição de modularidade dos deputados condicionada ao partido 
Tabela 3. Deputados com maiores homofilia condicionada a atributos

\begin{tabular}{|l|l|l|l|}
\hline- & $\mathbf{1}^{\mathbf{o}}$ & $\mathbf{2}^{\mathbf{o}}$ & $\mathbf{3}^{\mathbf{o}}$ \\
\hline Geral & BENEDITA DA SILVA & GIUSEPPE VECCI & DANRLEI HINTERHOLZ \\
\hline Escolaridade & ASSIS MELO $($ EM comp $)$ ) & HERCULANO PASSOS $($ EM comp. $)$ & JOÃO RODRIGUES $($ EM comp. $)$ \\
\hline Etnia & BENEDITA DA SILVA $($ negra $)$ & IZAQUE SILVA $($ parda $)$ & CARLOS ANDRADE $($ pardo $)$ \\
\hline Partido & JUNIOR MARRECA $($ PATRI $)$ & DELEGADO FRANCISCHINI $($ PATRI $)$ & JOÃO MORAES $($ PCdoB $)$ \\
\hline Sexo & SIMONE MORGADO $(F)$ & DÂMINA PEREIRA $(F)$ & BENEDITA DA SILVA $(F)$ \\
\hline Região & LÁZARO BOTELHO $(T O)$ & EZEQUIEL FONSECA $(M T)$ & CARLOS BEZERRA $(M T)$ \\
\hline
\end{tabular}

Os três deputados com menor homofilia partidária (Benedita da Silva, Reginaldo Lopes e Carlos Zarattini) pertencem ao PT, um dos partidos com maior homofilia média da rede. Dada essa observação, é possível inferir que mesmo em grupos significativamente coesos existem agentes independentes em suas articulações políticas, que muitas vezes levam mais em consideração em sua escolha de coautores a outras de suas características (atributos) cuja homofilia é maior.

Ainda, organizou-se na tabela 3 os três deputados com maior homofilia em cada um dos atributos analisados. A parlamentar com maior comportamento homofílico é Benedita da Silva, mulher negra que teve como coautoras apenas outras duas mulheres também negras (Rosangela Gomes e Tia Eron). Este resultado indica que, apesar de não ser válido para a Câmara dos Deputados como um todo, a proposição de Huddy [Huddy 2004] sobre maior coesão entre minorias se aplica em casos individuais. Não obstante, esse resultado corrobora com a hipótese de que características muito homofílicas de agentes individuais ( sexo e etnia) são capazes de sobrepujar características que são dominantes em influência na rede (partido).

\section{Conclusão e trabalhos futuros}

A proposição e aprovação de leis é provavelmente a tarefa mais importante da Câmara dos Deputados. Tendo em vista que as 513 cadeiras do congresso brasileiro são ocupadas por membros de 26 diferentes estados e 35 diferentes partidos (atualmente), além dos deputados serem de diferentes etnias e terem diferentes graus de escolaridade, a articulação política no processo de proposição e aprovação de leis se torna fundamental.

Este trabalho explorou a rede de coautoria dos projetos de lei para caracterizar a homofilia e assimetria inerente deste processo. Uma metodologia para quantificar a homofilia da rede e também do vértice (com relação a um atributo) foi desenvolvida e aplicada. A rede, construída a partir da fusão de dados públicos, com vértices representando deputados com 5 diferentes atributos e arestas com peso representando coautoria entre dois deputados, foi avaliada.

Dentre os principais resultados está a diferente homofilia entre os atributos da rede, com partido e estado exibindo a maior homofilia, e etnia e grau de escolaridade praticamente nenhuma homofilia. Outro importante descobrimento foi a alta assimetria na homofilia de diferentes valores de atributos. Por exemplo, ao considerar partido político, três partidos (de 27) possuem alta homofilia e mais de 20 possuem homofilia desprezível. Esta observação vai de encontro a teoria da homofilia das minorias, que diz que minorias tendem a possuir homofilia mais alta do que grupos majoritários.

Diferentes estudos podem ser elaborados como decorrência deste trabalho. Em particular, um melhor entendimento da ausência de homofilia entre as minorias, e a 
identificação de indivíduos mais influentes por conta de alta homofilia.

\section{Referências}

Baptista, V., Brito, F., Brasileiro, J., Duarte, A. N., Bezerra, E. P., Almeida, F., Lima, P., and Guimaraes, S. (2014). Uma ferramenta para analisar mudanças na coesao entre parlamentares em votaçoes nominais. In Brazilian Workshop on Social Network Analysis and Mining (BraSNAM).

Bursztyn, V. S., Nunes, M. G., and Figueiredo, D. R. (2016). How congressmen connect: analyzing voting and donation networks in the brazilian congress. In Brazilian Workshop on Social Network Analysis and Mining (BraSNAM).

Ferreira, C. H. G., de Sousa Matos, B., and Almeira, J. M. (2018). Analyzing dynamic ideological communities in congressional voting networks. In International Conference on Social Informatics, pages 257-273.

Fowler, J. H. (2006). Connecting the congress: A study of cosponsorship networks. Political Analysis, 14(4):456-487.

Huddy, L. (2004). Contrasting theoretical approaches to intergroup relations. Political Psychology, 25(6):947-967.

Kessler, D. and Krehbiel, K. (1996). Dynamics of cosponsorship. American Political Science Review, 90(3):555-566.

Levorato, M. and Frota, Y. (2017). Brazilian congress structural balance analysis. arXiv preprint arXiv:1609.00767.

Madan, A., Farrahi, K., Gatica-Perez, D., and Pentland, A. S. (2011). Pervasive sensing to model political opinions in face-to-face networks. In Inter. Conference on Pervasive Computing, pages 214-231.

Martin, S., Brown, W. M., Klavans, R., and Boyack, K. (2011). Openord: An open-source toolbox for large graph layout. SPIE Conference on Visualization and Data Analysis (VDA).

Miguel, L. F. (2003). Capital político e carreira eleitoral: algumas variáveis na eleição para o congresso brasileiro. Revista de Sociologia e Política, 20.

Miguel, L. F., Marques, D., and Machado, C. (2015). Capital familiar e carreira política no brasil: gênero, partido e região nas trajetórias para a câmara dos deputados. DADOS - Revista de Ciências Sociais, 58(3):721-747.

Newman, M. (2018). Networks. Oxford.

Nicolau, J. (2002). Como controlar o representante?: Considerações sobre as eleições para a câmara dos deputados no brasil. DADOS - Revista de Ciências Sociais, 45:219236.

Seder, J. P. and Oishi, S. (2009). Ethnic/racial homogeneity in college students' facebook friendship networks and subjective well-being. Journal of Research in Personality, 43(3):438 - 443.

Verona, L., Oliveira, J., da Cunha Hisse, J. V., and Campos, M. L. M. (2018). Metrics for network power based on castells' network theory of power: a case study on brazilian elections. Journal of Internet Services and Applications, 9(1):23. 